\title{
The temperature dependence of optical properties of tungsten in the visible and near-infrared domains: an experimental and theoretical study
}

\author{
Marco Minissale ${ }^{a, b}$, Cedric Pardanaud ${ }^{a}$, Régis Bisson $^{a}$, Laurent \\ Gallais $^{b}$ \\ ${ }^{a}$ Aix Marseille Univ, CNRS, PIIM, Marseille, France \\ ${ }^{b}$ Aix Marseille Univ, CRNS, Centrale Marseille, Institut Fresnel, Marseille, France \\ E-mail: marco.minissale@univ-amu.fr
}

\begin{abstract}
The knowledge of optical properties of tungsten at high temperatures is of crucial importance in fields such as nuclear fusion or aerospace applications. The optical properties of tungsten are well known at room temperature, but little has been done at temperatures comprised between $300 \mathrm{~K}$ and $1000 \mathrm{~K}$ in the visible and near-infrared domains. Here we investigate the temperature dependence of tungsten reflectivity from the ambient to high temperatures $(<1000 \mathrm{~K})$ in the $500-1050 \mathrm{~nm}$ spectral range, a region where interband transitions have a strong contribution. Experimental measurements, performed via a spectroscopic system coupled with a laser remote heating, show that the tungsten reflectivity increases with temperature and wavelength. We have described these dependences through a Fresnel and two Lorentz-Drude models. The Fresnel model reproduces accurately the experimental curve at a given temperature, but it is able to simulate the temperature dependency of reflectivity only thanks to an ad hoc choice of temperature formulae for the refractive indexes. Thus, a less empirical approach is preferred based on LorentzDrude models to describe the interaction of light and charge carriers in the solid. The first Lorentz-Drude model, which includes a temperature dependency on intraband transitions, fits experimental results only qualitatively. The second Lorentz-Drude model includes in addition a temperature dependency on interband transitions. It is able to reproduce quantitatively the experimental results, highlighting a non-trivial dependence of interband transitions as a function of temperature. Eventually, we use these temperature dependent Lorentz-Drude models to evaluate the total emissivity of tungsten from $300 \mathrm{~K}$ to $3500 \mathrm{~K}$ and we compare our experimental and theoretical findings with previous results.
\end{abstract}

\section{Introduction}

The study of light interaction with solids deals with how an electromagnetic field interacts with the localized electromagnetic field of the atoms of the solid. The light-matter interaction induces a change on the impinging electromagnetic field just as a temporary or permanent modification of the properties of matter. These changes depend basically 
on the strength and wavelength of the field of the light and the matter itself. Moreover, external influences on matter, such as temperature, pressure, and other external fields (electrical, magnetic), can change its interaction with light and its optical properties. An accurate knowledge of optical properties of matter is essential both to understand the fundamental principles underlying the physical mechanisms behind the laser-matter interaction and for a wide range of research fields and applications[1], i.e. photonics and plasmonics[2, 3, 4], optoelectronics[5, 6], nanomedicine [7], infrared pyrometry[8], laser heating[9], and laser damage resistance of optical components[10].

The optical properties of matter are usually described by using Lorentz-Drude models which treat electrons as damped particles, free (Drude term) or harmonically bound (Lorentz term), subject to external electric fields. By considering the temperature dependence of electron parameters (i.e. plasma frequency and damping), some works[11, 12] have succeeded to describe theoretically the dependence of optical properties as a function of temperature. We pinpoint that these temperature dependent Lorentz-Drude (TDLD) models work very well if the dielectric function is strongly dependent on intraband/Drude term; for example, optical properties of gold can be well described by a TDLD model for wavelengths $\lambda>500-600 \mathrm{~nm} \mathbf{( 2 . 4 8 - 2 . 0 6} \mathbf{e V})$, where the intraband term is dominant with respect to interband terms. On the contrary TDLD models start to fail when applied to more complex cases. The reason could lie on the complexity to explain the temperature dependence (TD, hereafter) of damping and optical phonons for complex band structures[13, 4]. Recently some experimental works have preferred a parametric approach to describe the TD of optical properties of matter $[14,15]$, by varying ad hoc TDLD parameters instead of using analytical formulae.

In this work we focus our attention on the TD of optical properties of tungsten. The first measurements of tungsten (W) reflectivity at room temperature dates back to $1917,[16]$ but the interest has remained broadly constant over time[17, 18, 6]. In particular the scientific interest for tungsten has grown recently thanks to its peculiar and useful propertiesł for fusion reactors[20, 21] and aerospace applications[22, 23]. This has lead to more and more accurate studies of the temperature dependence of optical properties of $\mathrm{W}[24,25,26,27,28,23]$. Nevertheless the TD of optical properties of $\mathrm{W}$ have been measured only for temperatures higher than $1000 \mathrm{~K}[26]$ and the agreement between different determinations is not always satisfactory. Optical constants of tungsten have been studied theoretically only at room temperature[18, 6], and, to the best of our knowledge, no scientific works deal with the TD of W from a theoretical point of view. As previously said, the reason could be that usual TDLD models only take into account the temperature dependence of the intraband/Drude term and thus it cannot simulate optical properties in a region where interband terms are also active. This is the case of $\mathrm{W}$ in the visible and near-infrared (NIR) domains, where interband transitions produce intense reflectivity drops.

$\ddagger$ high energy threshold for sputtering and low erosion under high heat loads[19]. 
The focus of the present work is twofold: to study experimentally and to theoretically describe the temperature dependence of optical properties of $\mathrm{W}$. We first present an experimental measurement of $\mathrm{W}$ reflectivity in the optical and NIR wavelength range (500-1050 nm) from room to high temperature (300-925 K). Secondly, we give a quantitative determination of the refractive index and the extinction coefficient of $\mathrm{W}$ and their temperature dependences, by using Fresnel equations. Subsequently, we describe two TDLD models developed in order to study the TD of free carriers and optical phonons in W. The first Lorentz-Drude model includes a temperature dependency on intraband transistions while the second includes in addition a temperature dependency on interband transitions. Eventually, we apply these models in order to estimate the total emissivity in a wide temperature range.

\section{Experimental setup}

Figure 1 shows the experimental setup developed to perform the experiments. A sample holder made in Macor (a machineable glass-ceramic) is located inside a vacuum chamber (base pressure $5 \times 10^{-3} \mathrm{mbar}$ ). Vacuum is needed in this type of measurement to prevent oxidation of the sample (see Supplementary Materials). The polycrystalline tungsten sample of tungsten $\left(7 \times 7 \times 0.3 \mathrm{~mm}^{3}\right)$ was provided by A.L.M.T. Corp (Japan) sample with a mirror-like polishing.

The sample can be heated by using a continuous wave High-Power Laser (AMTRON, HPL in Fig. 1) system that is based on fiber-coupled GaAs diode emitting at $805 \mathrm{~nm}$ (JENOPTIK Laser GmbH). The HPL beam can reach a maximum power of $200 \mathrm{~W}$ and it has a quasi-Gaussian profile. The beam diameter is $3 \mathrm{~mm}$ at the focal point (30 $\mathrm{cm})$. In our setup the heating system and the sample holder are decoupled since the HPL's head is placed outside the vacuum chamber. This represents a big advantage with respect to the case of coupled systems (e.g. heating resistor[29], heater coil[30], and thermal contact with heating ceramic plate[31]): firstly, samples can be changed rapidly and easily, secondly the heating system does not impose any constraints about sample sizes. The temperature of the sample is controlled ex-situ through an InfraRed Camera (FLIR, model A655sc, IRC in Fig. 1) allowing measurements of temperature in the 230-2300 K range and its spatial distribution on the sample. Calibration of the IRC has been performed by using a type $\mathrm{K}$ thermocouple. The IRC works in the $7.5-14 \mu \mathrm{m}$

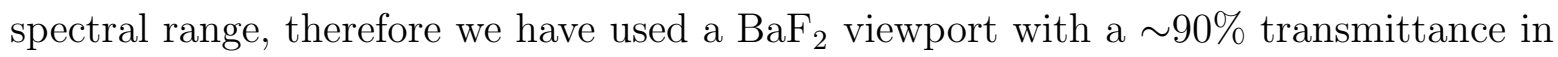
the considered spectral range. Via a visible camera (VC), we monitor continuously the position of the incident heating laser on the sample and the mechanical stability of the whole system (sample+sample holder).

Spectroscopic measurements are performed by using a Supercontinuum white Light source (LEUKOS, model SM-30-400, SL in Fig. 1) emitting $100 \mathrm{~mW}$ in the 400-2400 $\mathrm{nm}$ range. The SL source has a fibred collimated beam (diameter $<1 \mathrm{~mm}$ ) allowing the angle of incidence on the sample to be changed easily. Moreover using a collimated source allows all optics to be placed far away from the sample reducing considerably 


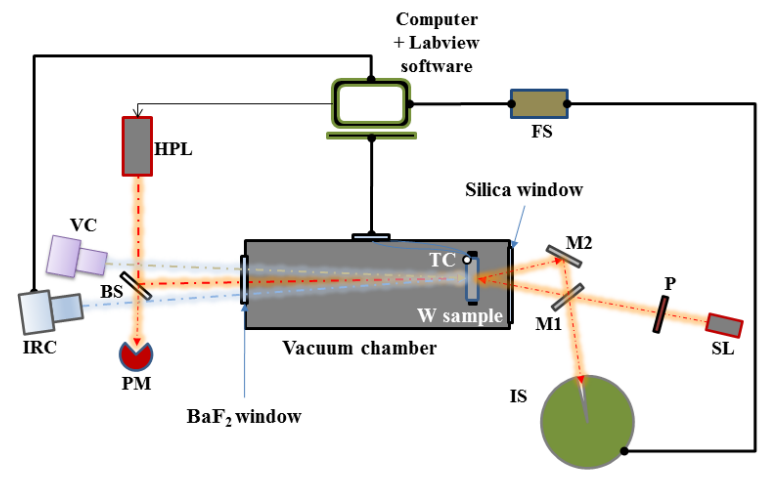

Figure 1. Schematic of the experimental setup: HPL, High Power Laser; BS, BeamSplitter; VC, Visible Camera; IRC, InfraRed Camera; PM, PowerMeter; TC, Thermocouple; M1 and M2, silver Mirrors; IS, Integrating Sphere; P, Polarizer; SL, Supercontinuum Laser.; FS, Fiber-optic Spectrometer

the detection of its black body radiation[32]. This is a critical issue in the case of high temperature measurements. The SL beam passes through a linear polarizer (Thorlabs, model LPVIS100-MP2, extinction ratio>10000:1) and reaches a removable reference silver mirror (Thorlabs, model PF05-03-P01) or the W sample. In the latter case, the SL beam crosses twice a silica window (before and after the $\mathrm{W}$ sample) and it is sent to a reference silver mirror. From the reference silver mirrors, the beam is sent to an integrating sphere made in Spectralon (LabSphere, IS in Fig. 1) and it reaches the fibred Spectrometer (Avantes, model AvaSpec-ULS2048L, FS in Fig. 1) through a $1000 \mu \mathrm{m}$ core fiber (Thorlabs, model M59L01). Since the spectrometer is sensitive in the 200-1100 $\mathrm{nm}$ wavelength range, it overlaps with the SL source only in the 400- $1100 \mathrm{~nm}$ region. Nevertheless we have performed spectroscopic measurements only in the 500-1050 nm where the best signal to noise ratio has been found.

The $\mathrm{W}$ reflectivity is measured by using the following equation

$$
R_{W}=\frac{S_{\text {meas }}-S_{\text {Dark }}}{S_{\text {ref }}-S_{\text {Dark }}} \frac{1}{T_{\text {Siw }}^{2}}
$$

where $\mathrm{S}_{\text {meas }}$ and $\mathrm{S}_{\text {ref }}$ are the spectra obtained by sending the SL beam on the W sample and to the reference mirror, respectively; $\mathrm{S}_{\text {Dark }}$ is the dark spectrum obtained with the SL beam switched off and the sample set to the desired temperature with the HPL heating source $\mathrm{ON} ; \mathrm{T}_{\text {Siw }}$ represents the transmission through the fused silica window (see Supplementary Materials for more details).

Reflectivity has been studied by varying three parameters:

- Polarization of the SL beam. Parallel (P) or perpendicular (S) to the plane of incidence.

- Sample temperature. Temperature is varied between 300 and $925 \mathrm{~K}$ by changing the HPL power sent on sample. 


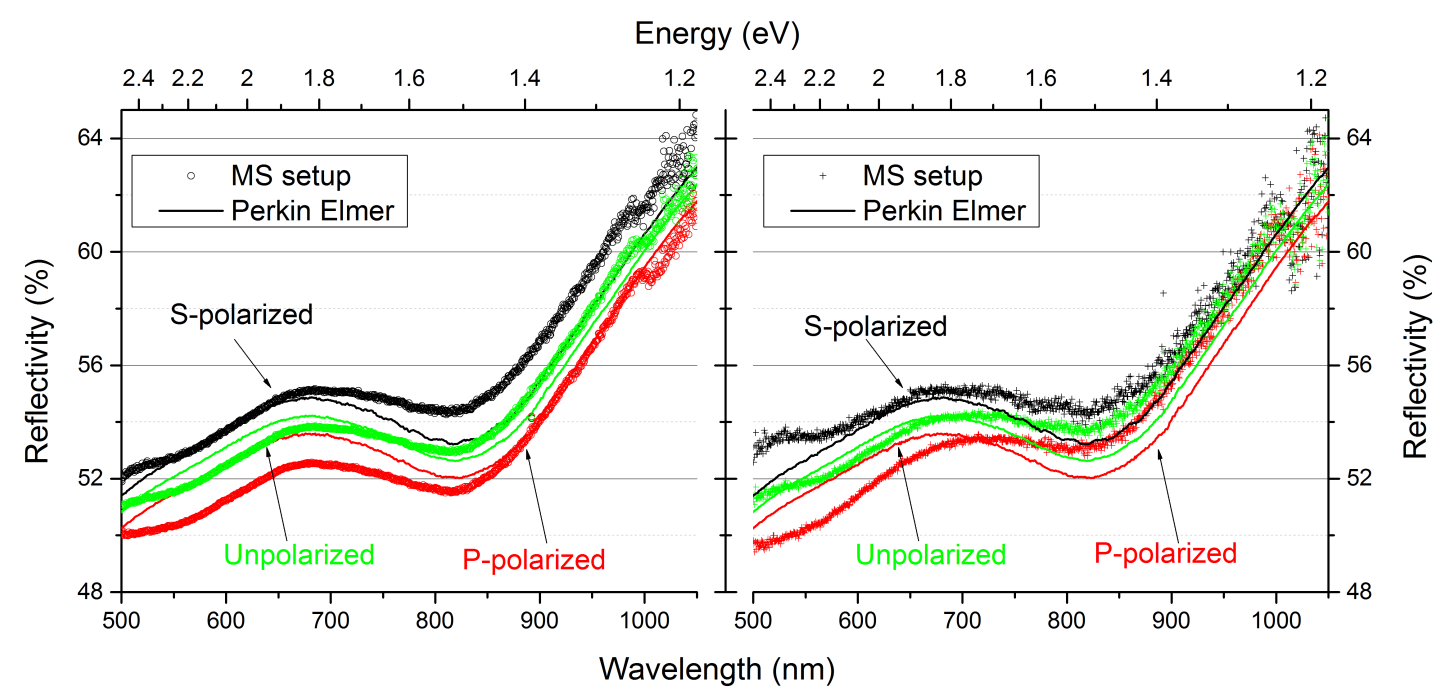

Figure 2. Reflectivity of tungsten for S-polarized (black), P-polarized (red), and unpolarized (green) light as a function of wavelength obtained with the MS setup described in section 2 (dots) and a Perkin Elmer spectrometer (solid lines). Spectra shown in left and right panels are performed without or with fused silica window in MS, respectively.

- Angle of incidence (AOI) of the SL beam. We have used three angles of incidence with respect to the normal: $20^{\circ}, 45^{\circ}, 50^{\circ}$

More details about experimental procedure and error bars are given in Supplementary Materials.

\section{Experimental results}

\subsection{Reflectivity: Polarization and angle dependence at room temperature}

We have performed measurements of tungsten reflectivity at room temperature by using two setups: the optical system described in section 2 (hereafter MS, main spectrometer) and a commercial UV/Vis/NIR spectrometer (Perkin Elmer LAMBDA 1050, hereafter PES). PES cannot be used at any AOI and in the case shown in Fig. 2, PES has been used with an incidence angle of 8 degrees while we used 20 degrees for MS (in both case the sample is held at room temperature). For this reason the data obtained with the two setups cannot be quantitatively compared, but can only give an idea of the accuracy of our optical system. As shown in equation 1, reflectivity measurements rely also on the measurement of transmission through the fused silica window. To measure the accuracy of MS, we have performed a first measurement without fused silica window. The results are shown in the left panel of Fig. 2. The order of magnitude of reflectivity in PES spectra (solid lines) is very similar to MS spectra: the maximum difference of reflectivity is $0.5 \%$ and it varies with wavelength. This amount can be considered as the best value of the MS (without window) accuracy for reflectivity measurements. The 


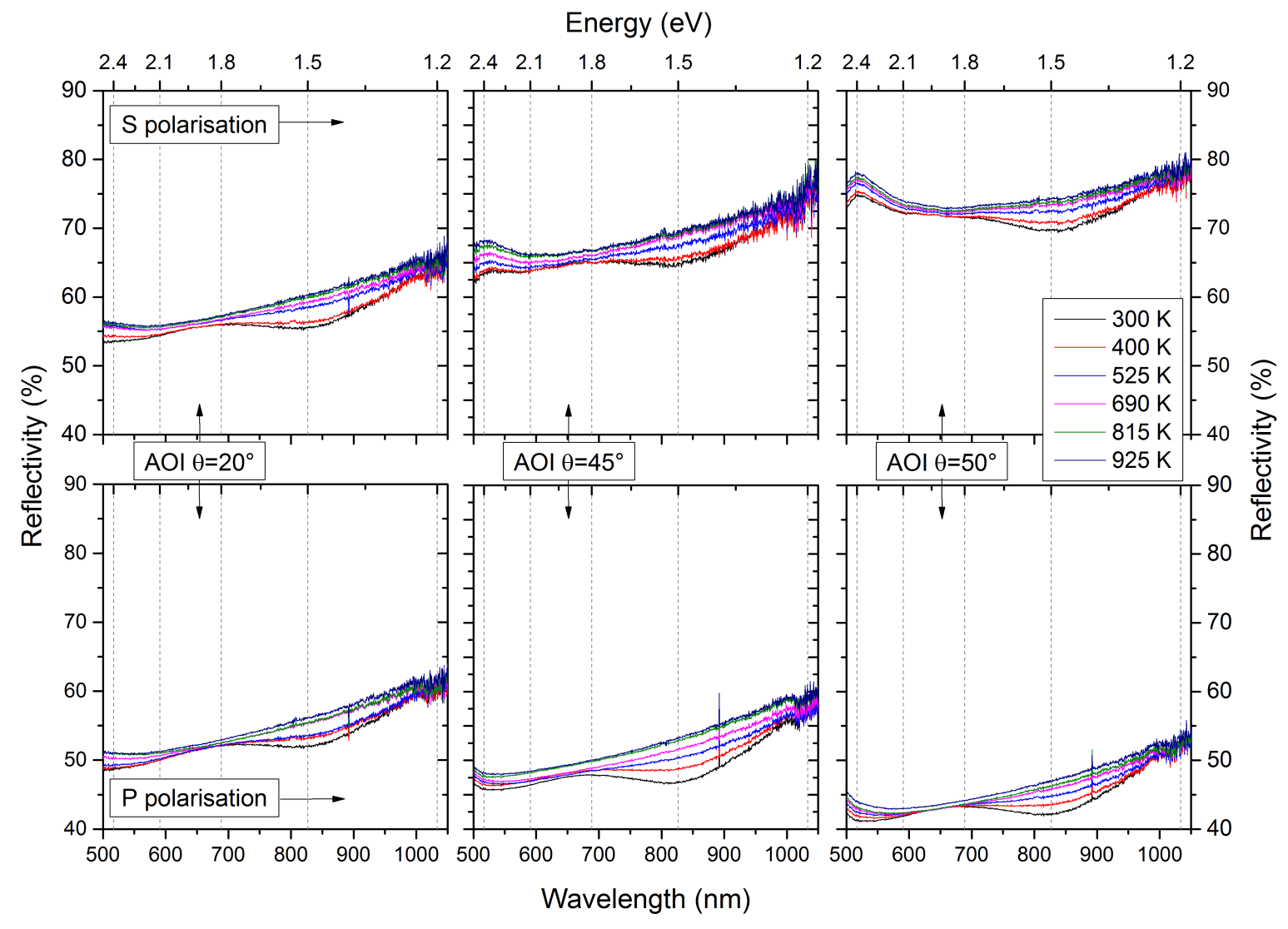

Figure 3. Reflectivity for tungsten of S (top panels) and P-polarized (bottom panels) light at six different temperatures $(300,400,525,690,815$, and $925 \mathrm{~K})$ for three incidence angles (from left to right, 20,45 , and $50^{\circ}$ ) as a function of wavelength.

observed difference could be due to the incidence angle: actually the most important effect of the AOI on reflectivity spectra is to accentuate the difference between $\mathrm{P}$ and $\mathrm{S}$ polarizations. In particular the higher is the incidence angle, the lower will be the reflectivity of P-polarized light (at least until the Brewster angle). On the contrary, reflectivity of S-polarized light will increase as a function of the AOI and unpolarized light will only slightly change. All these effects are partially observed by comparing the results of the two setups. We stress that our measurements are coherent with previous works with a maximum difference of $2 \%$ probably due to the different type of studied samples[16, 17].

All data presented hereafter have been obtained with the MS setup.

\subsection{Temperature dependence of reflectivity}

We present in Fig. 3 the spectral reflectivity of the tungsten sample held at different temperatures (300, 400, 525, 690, 815, and $925 \mathrm{~K}$ ) for $\mathrm{S}$ and $\mathrm{P}$ polarizations on top and bottom panels, respectively. We have studied tungsten reflectivity by changing the 
AOI of light, from the left to the right, to 20,45 and $50^{\circ}$. Table 1 sums up some of the results discussed in this section. In particular we list the reflectivity of unpolarized light obtained through the following equation

$$
R_{n o n-p o l}=\frac{R_{s}+R_{p}}{2}
$$

as a function of temperature and wavelength for the three AOIs used during experiments $\left(20,45\right.$, and $\left.50^{\circ}\right)$.

At first glance, one can see that an increase of the sample temperature produces an increase of the surface reflectivity. This growth is not linear both as a function of wavelength and temperature. In Fig. 4, we present the percentage variation (with respect to room temperature) of reflectivity as a function of temperature for three different wavelengths $(550,650$, and $850 \mathrm{~nm})$ in the case of S-polarized light with an AOI of $20^{\circ}$. The highest variation is at $850 \mathrm{~nm}$ where reflectivity has a percentage increase of $\sim 9 \%$ between 300 and $925 \mathrm{~K}$. In absolute value that means an increase from 55.9 to $60.8 \%$. At $650 \mathrm{~nm}$ there is a minimum of reflectivity variation with a percentage increase smaller than $2 \%$. For all other wavelengths, we have measured relative variations comprised between 2 and $9 \%$. Similar behaviors have been observed also for other AOIs and

Table 1. Temperature dependence of $\mathrm{W}$ reflectivity for different wavelengths.

\begin{tabular}{llcccccc}
\hline \multirow{2}{*}{$\begin{array}{l}\text { Wavelength } \\
\mathrm{nm}\end{array}$} & Energy & \multicolumn{5}{c}{ Reflectivity $^{a}$} \\
& $\mathrm{eV}$ & $300 \mathrm{~K}$ & $400 \mathrm{~K}$ & $525 \mathrm{~K}$ & $690 \mathrm{~K}$ & $815 \mathrm{~K}$ & $925 \mathrm{~K}$ \\
\hline 500 & 2.480 & $52.3(1.1) \S$ & $51.7(1.1)$ & $52.7(1.0)$ & $53.2(0.9)$ & $53.7(0.8)$ & $53.9(0.8)$ \\
550 & 2.255 & $52.8(0.8)$ & $51.8(0.8)$ & $52.5(0.9)$ & $52.8(0.8)$ & $53.3(0.8)$ & $53.5(0.7)$ \\
600 & 2.067 & $53.8(0.3)$ & $52.5(0.2)$ & $52.9(0.3)$ & $53.1(0.4)$ & $53.4(0.4)$ & $53.6(0.3)$ \\
650 & 1.908 & $54.9(0.4)$ & $53.5(0.4)$ & $53.7(0.4)$ & $53.9(0.4)$ & $54.1(0.5)$ & $54.4(0.4)$ \\
700 & 1.771 & $55.3(0.7)$ & $54.1(0.7)$ & $54.5(0.6)$ & $54.7(0.7)$ & $54.9(0.6)$ & $55.3(0.6)$ \\
750 & 1.653 & $55.3(1.2)$ & $54.4(1.2)$ & $55.1(1.1)$ & $55.7(1.2)$ & $56.0(1.3)$ & $56.3(1.3)$ \\
800 & 1.550 & $55.1(1.2)$ & $54.9(1.2)$ & $55.7(1.3)$ & $57.4(1.5)$ & $58.3(1.6)$ & $58.7(1.7)$ \\
850 & 1.459 & $55.5(1.2)$ & $55.2(1.1)$ & $56.5(1.2)$ & $57.9(1.2)$ & $58.3(1.3)$ & $58.8(1.3)$ \\
900 & 1.378 & $57.3(1.1)$ & $56.6(1.1)$ & $57.7(1.1)$ & $59.1(1.1)$ & $59.5(1.1)$ & $59.9(0.9)$ \\
950 & 1.305 & $59.9(1.2)$ & $58.7(1.2)$ & $59.5(1.1)$ & $60.6(1.2)$ & $61.1(1.2)$ & $61.6(1.1)$ \\
1000 & 1.240 & $62.7(1.0)$ & $61.1(1.0)$ & $61.7(1.0)$ & $62.2(1.0)$ & $62.8(1.0)$ & $63.4(1.2)$ \\
1050 & 1.181 & $64.1(0.9)$ & $61.8(1.1)$ & $62.3(1.2)$ & $63.1(0.9)$ & $63.4(1.3)$ & $64.0(1.3)$ \\
\hline
\end{tabular}

${ }^{a}$ Reflectivity (in \%) of unpolarized light at $\mathrm{AOI}=20^{\circ}$ obtained as a mean between $\mathrm{S}$ and P-polarized spectra.

for P-polarized light. The temperature dependence of all these spectra have a similar changing on spectrum shape as a common feature: the hill peaked around $700 \mathrm{~nm}$ and the well at $820 \mathrm{~nm}$ visible at room temperature become smoothed and disappear at 925 $\mathrm{K}$. In other words, spectra become more and more flattened with temperature increase as we will discuss extensively in section 4 from a theoretical point of view. 


\section{Models and Discussion}

In this section, we present the evaluation of the real refractive index $n$ and the extinction index $k$ and we describe two Lorentz-Drude (LD) models used to fit our experimental results. Firstly, we show how the computational procedure (both for refractive indexes evaluation and for LD models) is performed in the case of room-temperature data, and subsequently we expand the discussion to the temperature dependent data. We provide from one side the behavior of refractive index $n$ and the extinction coefficient $k$ as a function of temperature, and for the other side we discuss extensively temperature dependent Lorentz-Drude (TDLD) models, focusing our attention on the role of damping parameters. Finally, we show how TDLD models are able to predict optical properties (i.e. total emissivity) of $\mathrm{W}$ in a spectral range not used during present experiments.

\subsection{Temperature independent model}

4.1.1. Evaluation of $n$ and $k$ at room temperature from Fresnel reflectance equations The propagation properties of an electromagnetic field in a medium are commonly described by the dielectric function $\epsilon_{r}(\lambda)$ (also called relative permittivity), a dimensionless complex number defined as

$$
\hat{\epsilon_{r}}(\lambda)=\epsilon_{r}^{\prime}(\lambda)+i \cdot \epsilon_{r}^{\prime \prime}(\lambda)
$$

related to the refractive index by

$$
\hat{n}(\lambda)=\sqrt{\hat{\epsilon_{r}}(\lambda) \hat{\mu_{r}}(\lambda)}
$$

Also, the refractive index is generally a complex number depending on wavelength:

$$
\hat{n}(\lambda)=n(\lambda)+i \cdot k(\lambda)
$$

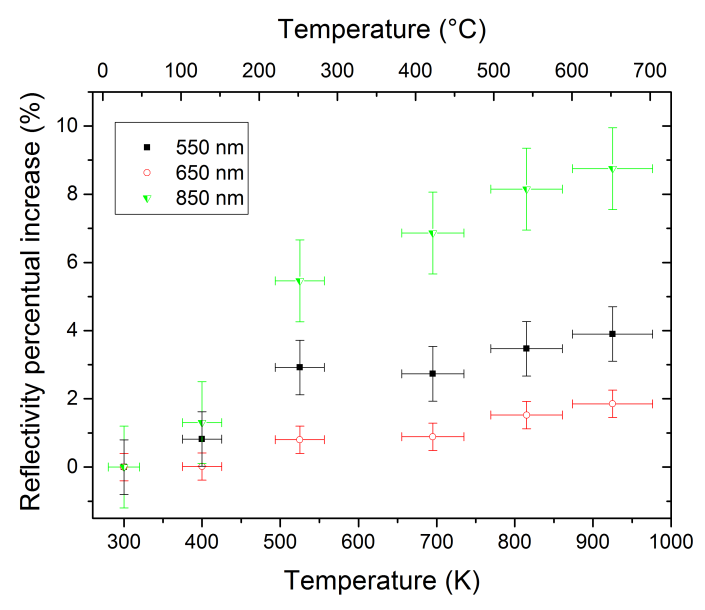

Figure 4. Reflectivity of tungsten for S-polarized light (incidence angle $=20^{\circ}$ ) at three wavelengths $(550,650$, and $850 \mathrm{~nm})$ as a function of temperature. 




Figure 5. Experimental (points) and theoretical (lines) reflectivity of tungsten at 900 $\mathrm{nm}$ as a function of the incidence angle of $\mathrm{S}$ (black), $\mathrm{P}$ (red) and unpolarized (green) light.

where $n$ is the real refractive index and it is related with the light propagation speed, while the imaginary part $k$, called extinction coefficient, defines the absorption by the medium. The refractive index $\hat{n}$ is related to the amplitudes, phases, and polarizations of the reflected and transmitted light that emerge when electromagnetic waves cross the interface between two media with different refractive index. This relation is described by the following equations (i.e. Fresnel equations, FE model)

$$
\begin{aligned}
& R_{s}=\left|\frac{n_{0} \cos [\theta]-n_{1} \sqrt{1-\left(\frac{n_{0}}{n_{1}} \sin [\theta]\right)^{2}}}{n_{0} \cos [\theta]+n_{1} \sqrt{1-\left(\frac{n_{0}}{n_{1}} \sin [\theta]\right)^{2}}}\right|^{2} \\
& R_{p}=\left|\frac{n_{0} \sqrt{1-\left(\frac{n_{0}}{n_{1}} \sin [\theta]\right)^{2}}-n_{1} \cos [\theta]}{n_{0} \sqrt{1-\left(\frac{n_{0}}{n_{1}} \sin [\theta]\right)^{2}}+n_{1} \cos [\theta]}\right|^{2},
\end{aligned}
$$

where $R_{s}$ and $R_{p}$ are the reflectivity of perpendicular and parallel polarizations, $n_{0}$ and $n_{1}$ are the refractive indexes of the two media, and $\theta$ is the AOI of light. These two equations can be used to predict the reflectivity of a medium by knowing its refractive index. Reciprocally, by measuring $R_{s}$ and $R_{p}$, it is possible to find the couple of values $(n, k)$. It can be done by performing an analytical inversion of Fresnel reflectance equations[33, 34]. Nevertheless, as claimed by Roy et al.[35], the resulting refractive index values are approximate and as a consequence the method is not suitable to find the complex index of refraction. For this reason, we prefer to evaluate numerically $n$ and $k$ as a function of wavelength. In order to find the best-fit of experimental data, we have varied $n$ and $k$ for each wavelength trying to minimize the Pearson chi square weighted through the error of experimental data $(\sigma)$ given below

$$
\chi^{2}=\sum_{\theta, p o l} \frac{1}{\sigma_{e x p}(\theta, p o l)}\left|\frac{R_{e x p}(\theta, p o l)-R_{t h}(\theta, p o l)}{R_{t h}(\theta, p o l)}\right|
$$

where $R_{\exp }(\theta, p o l)$ and $R_{t h}(\theta, p o l)$ are the experimental and theoretical reflectivities for a given angle and a given polarization, $\sigma_{\text {exp }}(\theta, p o l)$ is the experimental reflectivity error for a given angle and a given polarization. Figure 5 shows the comparison between 


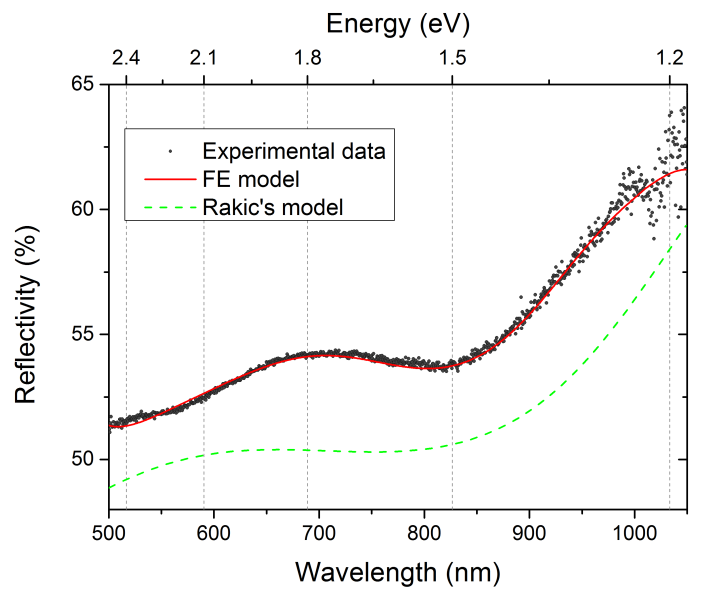

Figure 6. Experimental (dots) and theoretical (red line: Fresnel equations model; green dashed line: Rakic et al.[6]) reflectivity of tungsten at room temperature as a function of wavelength for unpolarized light at $\mathrm{AOI}=20^{\circ}$.

experimental and theoretical reflectivities at $900 \mathrm{~nm}$. Similar results have been obtained at other wavelengths. We have found a value of $\sim 74^{\circ}$ for the Brewster angle $\left(\theta_{b}\right)$ not far from the $77.5^{\circ}$ commonly used in the literature[6]. We stress that, depending on the wavelength, $\theta_{b}$ slightly varies with an average value of $75.2^{\circ}$. In Supplementary Materials, we provide a table with $n$ and $k$ minimizing equation 8 for wavelength in the 500-1050 nm domain (with a $10 \mathrm{~nm}$ step).

Figure 6 shows the simulated reflectivity spectrum obtained with the FE model (red line) compared to the experimental spectrum (dots). The FE model is also compared to the theoretical reflectivity spectrum from Rakic et al. [6].

We notice two main differences between the two theoretical lines obtained by fitting $n$ and $k$ (red and green line in Fig. 6). The first difference is the predicted amplitude of reflectivity. Theoretical evaluations of refractive indexes from Rakic et al.[6] underestimate reflectivity of tungsten by 2-3\%. We notice that the experimental data is consistent though two different apparatus (Figure 2). Thus, this problem of "offset" between the experimental data and Rakic's model could be related to the surface properties of tungsten since Rakic's work is solely a theoretical work, which did not take into account the presence of the native oxide layer that persist on tungsten up to 2400 $\mathrm{K}[36]$. We point out that we have analyzed the present sample surface oxidation state with Raman spectroscopy and did not find a significant change of oxidation throughout our measurements (see Supplementary Materials). Furthermore, we have checked with AFM and SEM the quality of the sample polishing. Nevertheless we will avoid dealing with the surface properties dependence of reflectivity since a more systematic study, lying outside the scope of this work, should be performed. The other main difference between the two simulated reflectivity spectra is their shape. As already said in the experimental section, reflectivity spectra of tungsten at room temperature presents two peculiar features: the hill peaked at $700 \mathrm{~nm}$ and the well at $820 \mathrm{~nm}$. These 
features exhibit their main evolution in temperature dependent spectra and they are not predicted by Rakic's refractive indexes. We pinpoint that, even if $n$ and $k$ of Rakic et al. are not able to simulate properly reflectivity of tungsten in our spectral domain, they are very powerful to predict $W$ reflectivity in a very broad wavelength range, from UV $(200 \mathrm{~nm})$ to far-IR $(12 \mu \mathrm{m})$. In any case, we will put aside $n$ and $k$ of Rakic et al. since the main goal of this work is to describe the optical properties of tungsten in the 500-1050 nm domain. We note that these two main differences between our model and Rakic's one could be the reasons behind the difference of $2.3^{\circ}$ found for $\theta_{b}$.

The FE model with an ad hoc choice of $n$ and $k$ parameters is able to reproduce accurately the experimental curve at a given temperature. However, it is not able to give the temperature dependency of reflectivity, i.e. one need to calculate ad hoc parameters every time for any sample temperature or use an empirical formulae (see section IV.B.1). Thus, it is desirable to use a less empirical approach where the interaction of light and charge carriers in the solid is physically described, which should allow to develop a temperature dependency based on physical ground. This goal can be achieved by using a Lorentz-Drude model as described in the next section and in section IV.B.2.

4.1.2. Lorentz-Drude model In this section, we present the Lorentz-Drude (hereafter LD) model developed to describe the electric permittivity of metals and to simulate the reflectivity of $\mathrm{W}$.

The optical properties of matter are usually described by using Lorentz-Drude models[37] which treat electrons as damped particles, free (Drude term) or harmonically bound (Lorentz term), subject to external electric fields.

In the LD model formalism intraband (Drude) and interband (Lorentz) transitions can be described by several oscillators through the following equation:

$$
\hat{\epsilon}_{r}=1-\frac{f_{0} \omega_{p}^{2}}{\omega\left(\omega-i \Gamma_{0}\right)}+\sum_{i=1}^{m} \frac{f_{i} \omega_{p}^{2}}{\omega_{i}^{2}-\omega^{2}+i \omega \Gamma_{i}},
$$

where $f_{0}$ and $\Gamma_{0}$ are the strength and the damping of the Drude oscillator, while $f_{i}$, $\omega_{i}$, and $\Gamma_{i}$ are the strength, the frequency, and the damping of the Lorentz oscillators, respectively. The reflectivity can be calculated by knowing the value of $\hat{\epsilon}_{r}$ through

$$
R=\left|\frac{\hat{\epsilon}_{r}^{1 / 2}-1}{\hat{\epsilon}_{r}^{1 / 2}+1}\right|^{2} .
$$

For the sake of clarity we stress that this law is strictly valid for a normal incident beam while we have used it to fit reflectivity curves at $\mathrm{AOI}=20^{\circ}$ for unpolarized light. Nevertheless, we point out that, in the case of $\mathrm{W}$, the difference between reflectivity curves at $\mathrm{AOIs}=0^{\circ}$ and $20^{\circ}$ should be $\sim 0.04 \%$, an amount not appreciable with our current experimental setup.

We have fitted our experimental data by using as initial parameters the $\omega_{i}, \Gamma_{i}$, and $f_{i}$ from Rakic et al.[6]. In Table 2, we list Rakic parameters as well as the parameters that 
Table 2. Values of the LD Model Parameters.

\begin{tabular}{llllllll}
\hline Oscillator & $i$ & \multicolumn{2}{c}{$\omega_{i}$} & \multicolumn{2}{c}{$\mathrm{f}_{i}$} & \multicolumn{2}{c}{$\Gamma_{i}$} \\
& & Rakic $^{a}$ & $\mathrm{TW}^{b}$ & Rakic & TW & Rakic & TW \\
\hline Drude & 0 & - & - & 0.206 & 0.275 & 0.064 & 0.05 \\
Lorentz & 1 & 1.004 & 0.94 & 0.054 & 0.06 & 0.530 & 0.72 \\
Lorentz & 2 & 1.917 & 1.86 & 0.166 & 0.19 & 1.281 & 1.33 \\
Lorentz & 3 & 3.580 & 3.35 & 0.706 & 0.75 & 3.332 & 2.44 \\
Lorentz & 4 & 7.498 & 7.70 & 2.509 & 2.39 & 5.836 & 2.80 \\
\hline
\end{tabular}

$\omega_{i}$ and $\Gamma_{i}$ are given in $\mathrm{eV}, \mathrm{f}_{i}$ are dimensionless.

${ }^{a}$ From Rakic et al. $[6],{ }^{b}$ This work

we found to simulate best our experimentally measured W reflectivity in the 500 - 1050 $\mathrm{nm}$ range at $300 \mathrm{~K}\left(\mathrm{~T}_{\text {room }}\right)$.

The requirement to obtain a proper fit in the $500-1050 \mathrm{~nm}$ spectral range pushes us to slightly change the parameters of Lorentz and Drude oscillators used in Rakic et al.[6]. The change of the strength of the Drude oscillator is responsible of an overall increase of reflectivity with respect to Rakic's evaluation. The other main difference between the two set of values is the damping values of Lorentz oscillators. The choice of the present values is able to reproduce the two peculiar features of tungsten reflectivity (i.e. the hill peaked at $700 \mathrm{~nm}$ and the well at $820 \mathrm{~nm}$ ) that are not predicted by the LD model of Rakic. We stress that each oscillator has an influence in the whole spectrum even if the influence is amplified at $\omega_{i}$.

\subsection{Temperature dependent models}

In this section, we present models developed to simulate the temperature dependence of tungsten reflectivity. In section 4.2.1, we discuss an empirical law reproducing the evolution of refractive indexes with $\mathrm{W}$ temperature, while in section 4.2 .2 , we present two temperature dependent Lorentz-Drude models. The physical ground of latter models allow, in section 4.3, to evaluate the temperature dependency of the total emissivity of $\mathrm{W}$ in different spectral ranges.

4.2.1. Evaluation of $n$ and $k$ as a function of temperature In section 4.1.1 we have treated the evaluation of $n$ and $k$ from reflectivity data at room temperature. By following the same procedure described in section 4.1.1, we have fitted the evolution of refractive indexes of tungsten as a function of its temperature. Clearly, refractive indexes depend here both on wavelength and temperature. Both $n$ and $k$ present a decreasing behaviour with temperature. By using two empirical laws, we have fitted the TD dependence of $n$ and $k$

$$
\begin{aligned}
& n_{\lambda, T}=n_{0}(\lambda)+n_{1}(\lambda) \cdot \exp \left(-n_{2}(\lambda) T\right) \\
& k_{\lambda, T}=k_{0}(\lambda)+k_{1}(\lambda) \cdot \exp \left(-k_{2}(\lambda) T\right)
\end{aligned}
$$


The temperature dependence of optical properties of tungsten

Table 3. Parameters ${ }^{a}$ used to fit the temperature dependence of the refractive index $n$ and the extinction coefficient $k$ of tungsten for different wavelengths.

\begin{tabular}{ll|ccc|ccc}
\hline $\begin{array}{l}\text { Wavelength } \\
\mathrm{nm}\end{array}$ & $\begin{array}{l}\text { Energy } \\
\mathrm{eV}\end{array}$ & $\mathrm{n}_{0}$ & $\mathrm{n}_{1}$ & $\mathrm{n}_{2}\left(10^{-3}\right)$ & $\mathrm{k}_{0}$ & $\mathrm{k}_{1}$ & $\mathrm{k}_{2}\left(10^{-3}\right)$ \\
\hline 500 & 2.480 & 0.427 & 10.557 & 5.787 & 0.904 & 3.590 & 0.211 \\
550 & 2.255 & 0.573 & 14.726 & 7.171 & 1.210 & 3.916 & 0.291 \\
600 & 2.067 & 0.615 & 15.113 & 7.294 & 1.237 & 3.960 & 0.285 \\
650 & 1.908 & 0.614 & 15.464 & 7.387 & 1.405 & 4.259 & 0.332 \\
700 & 1.771 & 0.637 & 18.020 & 7.988 & 1.582 & 4.851 & 0.408 \\
750 & 1.653 & 0.588 & 14.969 & 7.244 & 1.491 & 4.133 & 0.337 \\
800 & 1.550 & 0.573 & 13.443 & 6.792 & 1.365 & 3.613 & 0.267 \\
850 & 1.459 & 0.631 & 17.403 & 7.823 & 1.721 & 4.592 & 0.421 \\
900 & 1.378 & 0.586 & 22.017 & 8.773 & 1.694 & 5.839 & 0.479 \\
950 & 1.305 & 0.549 & 27.852 & 9.719 & 1.679 & 7.577 & 0.535 \\
1000 & 1.240 & 0.516 & 24.173 & 9.245 & 1.540 & 4.935 & 0.364 \\
1050 & 1.181 & 0.471 & 16.565 & 7.941 & 1.483 & 4.481 & 0.317 \\
\hline
\end{tabular}

${ }^{a}$ For $n$ and $k$ we have used the following laws, respectively

$n_{\lambda, T}=n_{0}(\lambda)+n_{1}(\lambda) \cdot \exp \left(-n_{2}(\lambda) T\right)$

$k_{\lambda, T}=k_{0}(\lambda)+k_{1}(\lambda) \cdot \exp \left(-k_{2}(\lambda) T\right)$

Table 3 shows the values of $n_{0,1,2}$ and $k_{0,1,2}$ parameters as a function of wavelength (with a step of $50 \mathrm{~nm}$ ). All parameters present a wavelength dependence that could be described through a second or third order polynomial. By averaging the values of $n$ and $k$ over all wavelengths $(500-1050 \mathrm{~nm})$ for each temperature, we can have a more general idea of the TD of refractive index, as shown in Fig. 7. The error bars represent standard deviations of $n$ and $k$ values in the considered wavelength range. By fitting mean values of $n$ and $k$ through equations $11 \mathrm{a}$ and $11 \mathrm{~b}$, we obtain

$$
\begin{aligned}
& n_{T}=0.562+14.272 \cdot \exp \left(-7.210^{-3} \cdot T\right) \\
& k_{T}=1.462+4.099 \cdot \exp \left(-3.310^{-3} \cdot T\right)
\end{aligned}
$$

We notice that the extinction coefficient $k$ decreases more slowly with respect to the real refractive index $n$ with a decay constant $\tau$ of 210 and $96 \mathrm{~K}$, respectively. We stress that these TD laws for $n$ and $k$ are strictly valid only in the temperature $(300<\mathrm{T}<925$ $\mathrm{K})$ and wavelength $(500<\lambda<1050 \mathrm{~nm})$ ranges in which they have been obtained.

4.2.2. Temperature dependent LD models Here, we present two modifications of the LD model presented in section 4.1.2 to take into account the temperature dependence. The main modification that has to be considered in equation 9 concerns damping factors. Actually, for a bulk metallic sample, the motion of electrons in the conduction band can undergo different damping mechanisms. The first source of damping for electrons is the electron-electron scattering $\Gamma_{e-e}$. This process is well known[38, 39] and Lawrence and Wilkins $[40,41]$ have described its frequency and temperature dependencies by using the following equation

$$
\Gamma_{e-e}=\frac{\pi^{3}}{12 \hbar E_{f}}\left[\left(k_{b} T\right)^{2}+\left(\frac{h \omega}{2 \pi}\right)^{2}\right]
$$


The temperature dependence of optical properties of tungsten

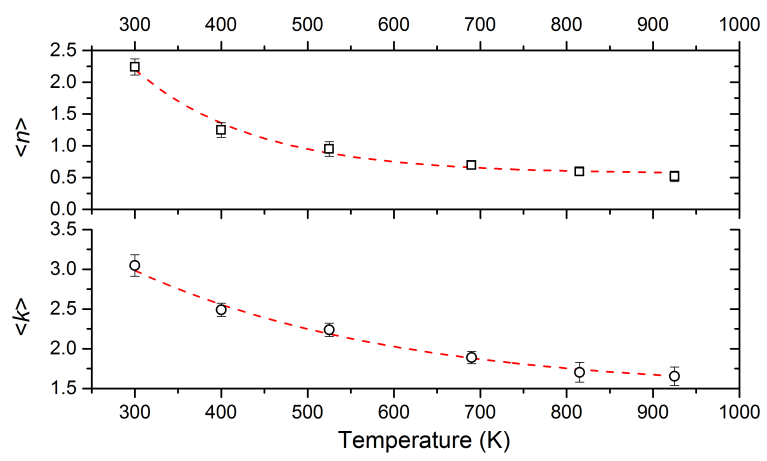

Figure 7. Temperature dependence of averaged (over 500-1050 nm) values of $n$ and $k$. Lines are plotted from equations $17 \mathrm{a}$ and $17 \mathrm{~b}$ (see text).
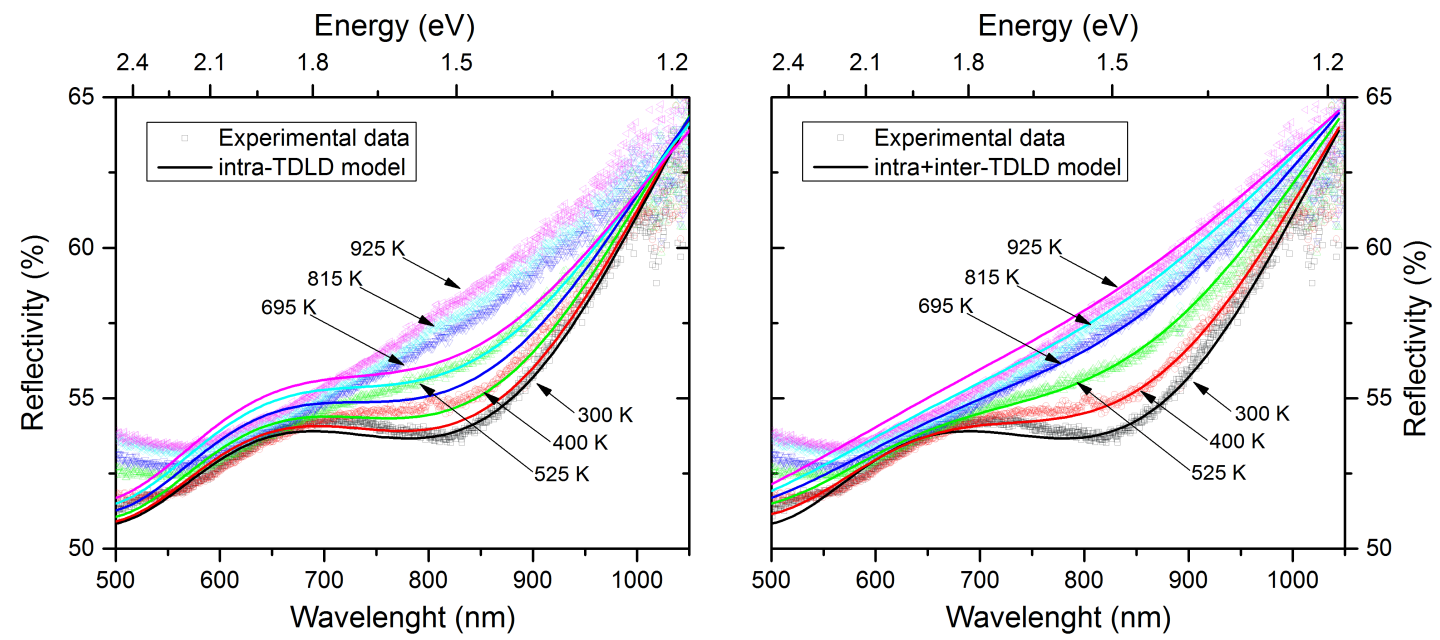

Figure 8. Measured and calculated reflectivity of unpolarized light at $20^{\circ}$ of AOI. Lines are obtained by using the intra-TDLD model (left panel) and the intra+interTDLD model (right panel) for six different temperatures $(300,400,525,695,815$, and $925 \mathrm{~K})$.

where $E_{f}$ is the Fermi energy, $k_{b}$ and $\hbar$ are the Boltzmann and Planck constant, respectively. We notice that in the optical spectral range the temperature dependent term turns to be negligible with respect to the frequency dependent counterpart[11, 12].

The second damping mechanism is the electron-phonon scattering $\Gamma_{e-p h}$. This term is the most important to describe the TD of damping. Its expression was derived for free electrons (intraband/Drude term) by Holstein [42, 43] and it is still extensively studied in different fields of research[4, 44]. A possible expression is given below (see Alabastri et al.[12] for more details)

$$
\Gamma_{e-p h}=\Gamma_{0}\left(\frac{2}{5}+4\left(\frac{T}{\theta}\right)^{5} \int_{0}^{\theta / T} \frac{z^{4} d z}{e^{z}-1}\right) .
$$

The TDLD model including a temperature dependence on the intraband/Drude term, i.e. the intra-TDLD model, is thus described by

$$
\hat{\epsilon}_{r}(T)=1-\frac{f_{0} \omega_{p}(T)^{2}}{\omega(\omega-i \Gamma(T))}+\sum_{i=1}^{m} \frac{f_{i} \omega_{p}(T)^{2}}{\omega_{i}^{2}-\omega^{2}+i \omega \Gamma_{i}} .
$$


Here, the temperature dependence of the plasma frequency includes a reduced electronic density due to the thermal volume expansion [45] and it is given by

$$
\omega_{p}(T)=\frac{\omega_{p}}{\sqrt{1+\gamma\left(T-T_{\text {room }}\right)}},
$$

where $\gamma$ is the thermal expansion coefficient of tungsten $\left(4.3 \times 10^{-6} \mathrm{~K}^{-1}\right)$. In order to reproduce the same damping parameter of the LD model at room temperature, we can write the damping parameter by subtracting the contribution calculated at room temperature as follows

$$
\Gamma(T)=\Gamma_{0}+\Gamma(T)-\Gamma\left(T_{\text {room }}\right) .
$$

The results of the intra-TDLD model are shown on the left panel in Fig. 8. In particular, we present the comparison of experimental (dots) and theoretical (solid lines) results, obtained for unpolarized light with an AOI of $20^{\circ}$ at three temperatures: 300, 400, 525, 690, 815, and $925 \mathrm{~K}$. As evident, the intra-TDLD model is not able to fit quantitatively the measured spectra, but it only succeeds to reproduce the overall behavior of reflectivity, i.e. its increase as a function of temperature. The difference of absolute reflectivity between the model and experiments varies as a function of wavelength. At room temperature, it is $0.1 \%$ (absolute value) at $\sim 850 \mathrm{~nm}$, while it reaches a maximum value of $\sim 2.4 \%$ for spectra taken at $925 \mathrm{~K}$.

As said in section 3.2, the most evident effect of the temperature increase is to flatten the hill peaked around $700 \mathrm{~nm}$ and the well at $820 \mathrm{~nm}$ present in room temperature spectra. In equation 15, we can note that the interband terms do not contain any temperature dependence and it could be the reason of the quantitative difference between theoretical and experimental spectra. Thus the flattening of the reflectivity spectrum observed experimentally could be due to a different response of Lorentz oscillators to a temperature increase. Different works[46, 47], studying gold nanoparticles, claimed that interband terms should have minimal or no effect on plasmon absorption with temperature since interband transition energies (typically of the order of the eV) are very high compared to vibrational energy $\left(\sim 10^{-2} \mathrm{eV}\right)$. Our results and some recent experimental works performed on different materials (ultrathin Au films[15], bis(ethylenedithio)tetrathiafulvalene[48], BSTS [49], LiInSe[14],) suggest a temperature dependence for interband terms contradicting the previous statement. Usually a parametric approach is preferred[48, 14] to fit the experimental observations, performed by an ad hoc change of Lorentz-Drude parameters. On the contrary, we preferred to reduce the number of free parameters, keeping the TD for Drude parameters. We have used a simple mechanical model[50] to consider the temperature dependence of Lorentz oscillators. In this TDLD model, so-called intra+inter-TDLD model, we consider that Lorentz oscillators experience a frictional force, modeled as a collisional resistance, increasing as a function of temperature. This is consistent with the fact that the probability of electron-phonon scattering increases as a function of temperature 
since, for a fixed frequency, the number of phonons increases following

$$
\left\langle n_{\text {phonons }}\right\rangle=\frac{1}{\exp \left(\frac{\hbar \omega}{k_{b} T}\right)-1}
$$

It is equivalent to say that atoms are vibrating more with the increase of temperature and they are more likely to be hit by bound electrons, increasing as a consequence the resistance of the metal. According to this model[50] and to equation 17, the damping will increase with temperature as:

$$
\Gamma_{i}(T)=\Gamma_{i}+\alpha\left(\sqrt{T}-\sqrt{T_{\text {room }}}\right)
$$

where $\alpha$ is a parameter expressed in $e V \cdot K^{-1 / 2}$. From the denominator of the Lorentz term in equation 15 , we find that

$$
\omega_{i}^{2}=\omega\left(\omega-i \Gamma_{i}\right) \propto-\sqrt{T}
$$

and the resonance frequency of Lorentz oscillators can be written as follows

$$
\omega_{i}^{2}(T)=\omega_{i}^{2}-\beta\left(\sqrt{T}-\sqrt{T_{\text {room }}}\right)
$$

where $\beta$ is a parameter expressed in $e V^{2} \cdot K^{-1 / 2}$. The intra+inter-TDLD model is thus described by

$$
\begin{gathered}
\hat{\epsilon}_{r}(T)=1-\frac{f_{0} \omega_{p}(T)^{2}}{\omega(\omega-i \Gamma(T))}+ \\
+\sum_{i=1}^{m} \frac{f_{i} \omega_{p}(T)^{2}}{\omega_{i}(T)^{2}-\omega^{2}+i \omega \Gamma_{i}(T)} .
\end{gathered}
$$

As evident from the right panel in Fig. 8, the intra+inter-TDLD model is able to reproduce quantitatively the flatten of reflectivity curves through the two supplementary TD Lorentz terms in equation 22. These curves have been obtained by using $\alpha=3.5 \cdot 10^{-2} \mathrm{eV} \cdot K^{-1 / 2}$ and $\beta=2.5 \cdot 10^{-3} \mathrm{eV}^{2} \cdot K^{-1 / 2}$. We list in Table 4 resonance frequencies and damping values (expressed in $\mathrm{eV}$ ) for Lorentz oscillators as a function of temperature. Therefore, the use of a frictional model to describe the temperature dependency of Lorentz oscillators in a Lorentz-Drude model seems to be an interesting subject of study for future works.

\subsection{From reflectivity to emissivity}

In the previous section, we have focused our attention on a Lorentz-Drude model which includes temperature dependences on both intraband and interband terms (intra+interTDLD) that is able to reproduce quantitatively reflectivity spectra. This new model can be used also to infer information about spectral emissivity. Actually, it is well known that for an opaque material (i.e. transmission=0) reflectivity and spectral emissivity are linked by the following relation

$$
\epsilon_{s}(\lambda)=1-R(\lambda)
$$


Table 4. Values of resonance frequencies and damping values calculated with equations 19 and 21 (expressed in $\mathrm{eV}$ ) for Lorentz oscillators at sample temperatures indicated in Fig. 8.

\begin{tabular}{lllllll}
\hline Oscillator & \multicolumn{7}{c}{ Temperature $(\mathrm{K})$} \\
Parameters & 300 & 400 & 525 & 695 & 815 & 925 \\
\hline$\omega_{\text {Lorent } z 1}$ & 0.940 & 0.934 & 0.926 & 0.919 & 0.914 & 0.909 \\
$\omega_{\text {Lorent } z 2}$ & 1.860 & 1.847 & 1.834 & 1.818 & 1.808 & 1.799 \\
$\omega_{\text {Lorent } z 3}$ & 3.350 & 3.327 & 3.303 & 3.274 & 3.256 & 3.240 \\
$\omega_{\text {Lorent } z 4}$ & 7.700 & 7.648 & 7.592 & 7.526 & 7.748 & 7.448 \\
\hline$\Gamma_{\text {Lorent } z 1}$ & 0.720 & 0.787 & 0.861 & 0.949 & 1.003 & 1.049 \\
$\Gamma_{\text {Lorent } z 2}$ & 1.330 & 1.455 & 1.590 & 3.212 & 1.853 & 1.939 \\
$\Gamma_{\text {Lorent } z 3}$ & 2.440 & 2.669 & 2.917 & 1.751 & 3.399 & 3.558 \\
$\Gamma_{\text {Lorentz } 4}$ & 2.800 & 3.062 & 3.348 & 3.686 & 3.900 & 4.083 \\
\hline
\end{tabular}

In turn, it is possible to find the total emissivity from spectral emissivity through the following equation

$$
\epsilon_{t o t}=\frac{\int_{\lambda_{1}}^{\lambda_{2}} \epsilon_{s}(\lambda) \lambda^{-5} \cdot \exp \left[\frac{c 2}{\lambda T}\right]^{-1} d \lambda}{\int_{\lambda_{1}}^{\lambda_{2}} \lambda^{-5} \cdot \exp \left[\frac{c 2}{\lambda T}\right]^{-1} d \lambda}
$$

where $\mathrm{c} 2$ is the Planck's second radiation constant, $\lambda_{1}$ and $\lambda_{2}$ are the limits of integration. By using equations 23 and 24, we have chosen three different couples of integration limits: two $(0.6<\lambda<2.8 \mu \mathrm{m}$ and $0.6<\lambda<40 \mu \mathrm{m})$ to perform a direct comparison with reflectivity data of $\mathrm{W}$ annealed at $1700 \mathrm{~K}$ from Brodu et al.[23] and a third one $(0.4<\lambda<300 \mu \mathrm{m})$ to do a direct comparison with hemispherical total emissivity measurements from Matsmuto et al.[26], Worthing[51], and Rudkin[52]. To evaluate the total emissivity, we have used both the intra-TDLD model and the intra+inter-TDLD model (solid lines and full dots in Fig. 9, respectively).

Total emissivity is proportional to the integral of $\epsilon_{s}$ over the considered spectral range, and thus it is inversely proportional to the reflectivity. This means that $\epsilon_{\text {tot }}$ evaluated in the visible and near-IR will be higher than $\epsilon_{t o t}$ in the IR or far-IR. This is confirmed by looking to solid red line or full red circles $(0.6-2.8 \mu \mathrm{m})$ and solid green line or full green triangles $(0.6-40 \mu \mathrm{m})$ in Fig. 9. Moreover, we see that $\epsilon_{t o t}(0.6-40 \mu \mathrm{m})$ is in good agreement with results of Brodu et al. 2015 (open green triangles). On the contrary, our data do not follow the same behavior of $\epsilon_{t o t}(0.6-2.8 \mu \mathrm{m})$ from Brodu et al. 2015 (open red circles). Surprisingly, these data do not present any temperature dependence, presenting an almost constant value of $\epsilon_{\text {tot }} \sim 0.4$ in the 1000-1700 K temperature range, and contradict our experimental results. We stress that our reflectivity measurements did not present any hysteresis, i.e. measurements performed at $300 \mathrm{~K}$ are identical before and after measurements at $925 \mathrm{~K}$, consistent with the negligible oxidation of our samples (see Supplementary Materials). The discrepancy of our results with Brodu et al. results for this wavelength range is therefore not understood for the moment.

Figure 9 also shows data from Matsumoto et al.[26] (squares), Worthing[51] (pentagons) 


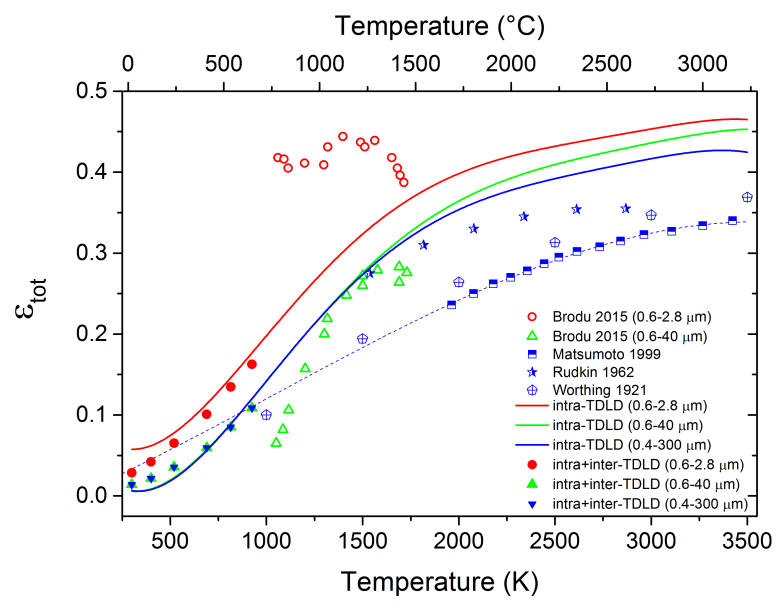

Figure 9. Calculated or measured total emissivity of tungsten from different references. Solid lines and full dots have been obtained for different spectral ranges through the intra-TDLD and the intra+inter-TDLD models, respectively. Blue dots are taken from Matsumoto et al.[26] (squares), Worthing[51] (pentagons) and Rudkin[52] (stars). Blue dashed curve is a fit of data from Matsumoto et al.[26] Open circles and triangles are taken from Brodu et al.[23]

and Rudkin[52] (stars). These data cannot be easily compared to our data since the physical quantity measured in these works is slightly different than the total emissivity as defined in equation 24. Emissivity measurement in these works is based on the heat balance of the heated sample in a brief steady-state period. In other words, at temperatures higher than $1500 \mathrm{~K}$, heat loss from sample happens essentially by thermal radiation and the electrical power imparted to the sample to heat it is equal to the power loss from the sample. So, by measuring the current and voltage drop the across sample, it is possible to derive emissivity from the Stephan-Boltzmann law. This type of measurement is not dependent on the wavelength range, but it considers the whole black body radiation. For this reason we have calculated the emissivity through the intra-TDLD and the intra+inter-TDLD models by extending the spectral range from $0.4 \mu \mathrm{m}$ to $300 \mu \mathrm{m}$ (solid blue line and blue full triangles). Results obtained through the intra-TDLD and the intra+inter-TDLD models are in good agreement with the fit of data from Matsumoto et al. [26] at low temperatures (below $1000 \mathrm{~K}$ ). On the other hand our calculations seem to overestimate $\epsilon_{\text {tot }}(0.4-300 \mu \mathrm{m})$ with respect to previous results at high temperatures $(2500-3500 \mathrm{~K})$. We may explain this difference by considering that in this temperature range the solid-liquid phase transition could activate different physical mechanisms (not considered in our models) and thus induce a different light-matter interaction.

\section{Summary and outlook}

In the present work, optical properties of tungsten has been studied as a function of its temperature. We have shown experimentally that the tungsten reflectivity shows a 
temperature dependence in a spectral range $(500-1050 \mathrm{~nm})$ and in a temperature domain (300-925 K) never studied in the literature until now. The temperature increase induces a non-linear reflectivity variation as a function of wavelength: we have measured between 300 and $925 \mathrm{~K}$ relative variations comprised between $2 \%$ (at $650 \mathrm{~nm}$ ) and $9 \%$ (at $850 \mathrm{~nm}$ ).

We have developed two different models to simulate the observed temperature dependence of tungsten optical properties. The first one, based on Fresnel equations, is able to reproduce accurately the experimental curve at a given temperature, but it is able to simulate the temperature dependency of reflectivity only thanks to an ad hoc choice of temperature formulae for refractive indexes. The second model is based on the LorentzDrude formalism and uses a less empirical approach to describe the interaction of light and charge carriers in the solid. A first version of the temperature dependent LorentzDrude model includes the temperature dependence only in the Drude term (intraband transitions) and it is able to reproduce qualitatively the increase of $\mathrm{W}$ reflectivity as a function of temperature. A second version of the model has been developed which describes the temperature dependence in the Lorentz terms (interband transitions) as a frictional force exerted on bound electrons. This model shows a better agreement with experimental results than the first version. Thanks to quantitative agreement between our reflectivity measurements and this new Lorentz-Drude model, we have shown that interband transitions should present a temperature dependence. We stress that this new result is not in disagreement with literature. Actually, previous experimental and theoretical works have studied temperature dependence of optical properties of metals in a spectral range where only intraband transitions are strongly active (e.g. Au in the visible domain, or $\mathrm{W}$ in the IR domain), while our work deals with a system (W/visible) where interband transitions can be probed.

Once the model has been validated in the spectral and temperature domain used for experiments, it has been extended up to $300 \mu \mathrm{m}$ and $3500 \mathrm{~K}$ to evaluate the total emissivity of W. Our theoretical results are in good agreement with emissivity measurements performed at high temperature (from 1000 to $2500 \mathrm{~K}$ ) and for the first time deal with the temperature behaviour of total emissivity at temperatures below $1000 \mathrm{~K}$.

Our results are particularly interesting for fusion[20, 21] and aerospace communities[22, 23] since tungsten, thanks to its peculiar and useful properties, is suitable for environments where high photon and particles fluxes are present. For example tungsten has been proposed for use in spacecraft sent near the sun (e.g. Solar Probe Plus mission by NASA) or in the divertor of future fusion devices (e.g. ITER). In the case of ITER, the working temperature range of the divertor starts around $345 \mathrm{~K}$ making our study fully relevant. Additionally, a better knowledge of tungsten properties (optical and mechanical) is needed in order to prevent security issues or failing of aerospace missions $[53,54,55,56]$.

We consider in the future to enlarge these experimental studies to the IR spectral 
range and for temperatures up to $2000 \mathrm{~K}$. To this end, we are developing a UHV setup to prevent oxidation of the sample for temperature above $1000 \mathrm{~K}$. This development will enable the simultaneous study of the influence of oxidation state, morphology, and temperature on optical properties of different refractory metals, such as tungsten or molybdenum.

\section{Acknowledgments}

This work has been carried out thanks to the support of the A*MIDEX project (n ANR-11-IDEX-0001-02) funded by the "Investissements d'Avenir" French Government program, managed by the French National Research agency (ANR). MM thanks A. Alabastri for fruitful discussion about the temperature dependent Lorentz Drude model.

[1] Thèye M L 1970 Physical Review B 2(8) 3060-3078 URL http://link.aps.org/doi/10.1103/ PhysRevB.2.3060

[2] Blanchard R, Boriskina S V, Genevet P, Kats M A, Tetienne J P, Yu N, Scully M O, Negro L D and Capasso F 2011 Opt. Express 19 22113-22124 URL http://www.opticsexpress. org/abstract. cfm?URI=oe-19-22-22113

[3] Neubrech F, Weber D, Katzmann J, Huck C, Toma A, Di Fabrizio E, Pucci A and Hartling T 2012 ACS Nano 6 7326-7332

[4] Lindenfeld Z and Lifshitz R 2013 Physical Review B 87 ISSN 1098-0121, 1550-235X URL http://link.aps.org/doi/10.1103/PhysRevB.87.085448

[5] Du G, Stair K A, Devane G, Zhang J, Chang R P H, White C W, Li X, Wang Z and Liu Y 1996 Semiconductor Science and Technology 111734 URL http://stacks .iop.org/0268-1242/11/ $i=11 / a=015$

[6] Rakic A, Djurisic A B, Elazar J M and Majewski M L 1998 Applied Optics 37 5271-5283

[7] Brullot W, Valev V and Verbiest 2012 Nanomed. Nanotechnol. Biol. Med. 8559568

[8] Imrie D A 2009 Photonics Spectra 43 49-52

[9] Prokhorov A, Konov V, Ursu I and Mihailescu N 1990 Laser Heating of Metals (CRC Press, Series in Optics and Optoelectronics)

[10] Ristau D 2015 Laser-Induced Damage in Optical Materials (CRC Press)

[11] McKay J and Rayne J 1976 Physical Review B 13 673-685

[12] Alabastri A, Tuccio S, Giugni A, Toma A, Liberale C, Das G, De Angelis F, Di Fabrizio E and Proietti Zaccaria R 2013 Materials 6 4879-4910

[13] Lin Z, Zhigilei L V and Celli V 2008 Physical Review B 77 ISSN 1098-0121, 1550-235X URL http://link.aps.org/doi/10.1103/PhysRevB.77.075133

[14] Liang Q, Wang S, Tao X and Dekorsy T 2015 Physical Review B 92 ISSN 1098-0121, 1550-235X URL http://link.aps.org/doi/10.1103/PhysRevB.92.144303

[15] Brandt T, Hovel M, Gompf B and Dressel M 2008 Physical Review B 78 ISSN 1098-0121, 1550235X URL http://link.aps.org/doi/10.1103/PhysRevB.78.205409

[16] Coblentz W W and Emerson W 1917 Reflecting power of tungsten and stellite Tech. rep. Bulletin of the Bureau of Standards

[17] Weaver J, Olson C and Lynch D 1975 Physical Review B 45 1293-1297

[18] Ordal M A, Bell R J, Alexander R W, Long Jr L L and Querry M R 1985 Applied Optics 24 4493-4499

[19] Bolt H, Barabash V, Federici G, Linke J, Loarte A, Roth J and Sato K 2002 Journal of Nuclear Materials 307 43-52

[20] Yoshida N 1999 Journal of Nuclear Material 266197206 
[21] Abernethy R 2016 Materials Science and Technology 1-12 ISSN 0267-0836, 1743-2847 URL https://www.tandfonline.com/doi/full/10.1080/02670836.2016.1185260

[22] Freeman R, Rigby F, Doerr S, Grimes L E and Ward D 1998 Reflectance of laser-damaged spacecraft thermal control materials SPIE Conference on High-Power Laser Ablation

[23] Brodu E, Balat-Pichelin M, Sans J L and Kasper J 2015 Acta Materialia 84 305-316 ISSN 13596454 URL http://linkinghub.elsevier.com/retrieve/pii/S1359645414008143

[24] Ujihara K 1972 Journal of Applied Physics 43 2376-2383

[25] Aksyutov N 1977 Zhurnal Prikladnoi Spektroskopii 26 914-918

[26] Matsumoto T, Cezairliyan A and Basak D 1999 International journal of thermophysics 20 943-952 URL http://link.springer.com/article/10.1023/A:1022699622719

[27] Cagran C, Pottlacher G, Rink M and Bauer W 2005 International Journal of Thermophysics 26 1001-1015 ISSN 0195-928X, 1572-9567 URL http://link.springer.com/10.1007/ s10765-005-6680-1

[28] Borghesani A and Carugno G 2015 Temperature dependent polarization of the thermal radiation emitted by thin, hot tungsten wires arXiv

[29] Yao H, Snyder P G and Woollam J A 1991 Journal of applied physics 70 3261-3267 URL http://scitation.aip.org/content/aip/journal/jap/70/6/10.1063/1.349285

[30] Vinod E, Naik R, Faiyas A, Ganesan R and Sangunni K 2010 Journal of Non-Crystalline Solids 356 2172-2174 ISSN 00223093 URL http://linkinghub.elsevier.com/retrieve/ $\mathrm{pii} / \mathrm{S} 0022309310004473$

[31] de Silans T P, Laliotis A, Maurin I, Gorza M P, Segundo P C, Ducloy M and Bloch D 2014 Laser Physics 24074009 URL http://stacks.iop.org/1555-6611/24/i=7/a=074009

[32] Minissale M, Bisson R and Gallais L 2016 Laser-induced damage in optical materials Proc. SPIE vol $10014 \mathrm{p} 100141 \mathrm{Q}$

[33] Querry M R 1969 Journal of the Optical Society of America $\mathbf{5 9} 876$

[34] Armaly B F, Ochoa J G and Look D C 1972 Applied Optics 11 2907-2910

[35] Roy S, Bang S Y, Modest M F and Stubican V S 1993 Applied Optics 32 3550-3558 URL https://www. osapublishing.org/abstract.cfm?uri=ao-32-19-3550

[36] Engel T, Niehus H and Bauer E 1975 Surface Science 52237

[37] Fox M 2010 Optical Properties of Solids 2nd ed (Oxford Master Series in Physics)

[38] Gurzhi R, Ya Azbel M and Lin X 1963 Solid State Physics 5 759-768

[39] Gurzhi R and Kaganov M 1966 Journal of Experimental and Theoretical Physics 3 654-656

[40] Lawrence W and Wilkins J 1973 Physical Review B 7 2317-2332

[41] Lawrence W 1976 Physical Review B 13 5316-5319

[42] Holstein T 1954 Physical Review 96 535-536

[43] Holstein T 1964 Annals of Physics 29 410-535

[44] Raju C N and Chatterjee A 2016 Scientific Reports 618511 ISSN 2045-2322 URL http: //www.nature.com/articles/srep18511

[45] Bouillard J S G, Dickson W, OConnor D P, Wurtz G A and Zayats A V 2012 Nano Letters 12 1561-1565 pMID: 22339644 (Preprint http://dx.doi.org/10.1021/nl204420s) URL http: //dx.doi.org/10.1021/nl204420s

[46] Etchegoin P G, Le Ru E C and Meyer M 2006 Journal of Chemical Physics 125164705

[47] Maurya M R and Toutam V 2016 The Journal of Physical Chemistry C 120 19316-19321 ISSN 1932-7447, 1932-7455 URL http://pubs.acs.org/doi/abs/10.1021/acs.jpcc.6b05847

[48] Olejniczak I, Frackowiak A, Swietlik R, Prokhorova T G and Yagubskii E B 2013 ChemPhysChem 14 3925-3935 ISSN 14394235 URL http://doi.wiley.com/10.1002/cphc.201300754

[49] Tang C S, Xia B, Zou X, Chen S, Ou H W, Wang L, Rusydi A, Zhu J X and Chia E E M 2013 Scientific Reports 3 ISSN 2045-2322 URL http://www.nature.com/articles/srep03513

[50] Yokozeki A, Kasprzak D J and Shiflett M B 2007 Physical Chemistry Chemical Physics 95018 ISSN 1463-9076, 1463-9084 URL http://xlink.rsc.org/?DOI=b706497g

[51] Worthing A G and Forsythe W E 1921 Physical Review 18144 
[52] Rudkin R L, Parker W J and Jenkins R J 1962 Temperature: Its Measurement and Control in Science and Industry vol 3 (Reinhold, New York) pp 523-534

[53] Eren B, Marot L, Langer M, Steiner R, Wisse M, Mathys D and Meyer E 2011 Nuclear Fusion 51 103025 ISSN 0029-5515, 1741-4326 URL http://stacks.iop.org/0029-5515/51/i=10/a= 103025?key=crossref . 8c733e83880201a27c3519c839a05e05

[54] Eren B, Marot L, Wisse M, Mathys D, Joanny M, Travère J M, Steiner R and Meyer E 2013 Journal of Nuclear Materials 438 S852-S855 ISSN 00223115 URL http://linkinghub. elsevier.com/ retrieve/pii/S002231151300192X

[55] Moser L, Steiner R, Leipold F, Reichle R, Marot L and Meyer E 2015 Journal of Nuclear Materials 463 940-943 ISSN 00223115 URL http://linkinghub.elsevier.com/retrieve/ $\mathrm{pii} / \mathrm{S} 0022311514008915$

[56] Moser L, Marot L, Eren B, Steiner R, Mathys D, Leipold F, Reichle R and Meyer E 2015 Nuclear Fusion 55063020 URL http://stacks.iop.org/0029-5515/55/i=6/a=063020 